\title{
Quantum gravitational decoherence of matter waves
}

\author{
Charles H-T Wang ${ }^{1,2, a}$, Robert Bingham ${ }^{2,3, b}$ and J Tito Mendonça ${ }^{4,2, c}$ \\ ${ }^{1}$ School of Engineering and Physical Sciences, \\ University of Aberdeen, King's College, Aberdeen AB24 3UE, UK \\ ${ }^{2}$ Rutherford Appleton Laboratory, Chilton, Didcot, Oxon OX11 0QX, UK \\ ${ }^{3}$ (SUPA) Department of Physics, \\ University of Strathclyde, Glasgow G4 0NG, UK \\ ${ }^{4}$ CFP, Instituto Superior Tecnico, 1049-001 Lisboa, Portugal \\ ${ }^{a}$ c.wang@abdn.ac.uk, ${ }^{b}$ r.bingham@rl.ac.uk, ${ }^{c}$ titomend@ist.utl.pt \\ PACS numbers: 04.60.Ds, 03.65.Yz, 03.75.-b
}

\begin{abstract}
One of the biggest unsolved problems in physics is the unification of quantum mechanics and general relativity. The lack of experimental guidance has made the issue extremely evasive, though various attempts have been made to relate the loss of matter wave coherence to quantum spacetime fluctuations. We present a new approach to the gravitational decoherence near the Planck scale, made possible by recently discovered conformal structure of canonical gravity. This leads to a gravitational analogue of the Brownian motion whose correlation length is given by the Planck length up to a scaling factor. With input from recent matter wave experiments, we show that the minimum value of this factor to be well within the expected range for quantum gravity theories. This suggests that the sensitivities of advanced matter wave interferometers may be approaching the fundamental level due to quantum spacetime fluctuations and that investigating Planck scale physics using matter wave interferometry may become a reality in the near future.
\end{abstract}

Physics on the large scale is based on Einstein's theory of general relativity (GR), which interprets gravity as the curvature of spacetime. Despite its tremendous success as an isolated theory of gravity, GR has proved problematic in integration with physics as a whole, in particular the physics of the very small governed by quantum mechanics. There can be no unification of physics, which does not include them both. Superstring theory 1] and its recent extension to the more general theory of branes is a popular candidate, but the links with experiment are very tenuous. Loop quantum gravity 2, 3 attempts to quantize GR without unification, and has so far received no obvious experimental verification.

One hundred years ago, when Planck introduced the constant named after him, he also introduced the Planck scales, which combined this constant with the velocity of light $c$ and Newton's gravitational constant $G$ to give the fundamental Planck time $T_{\text {Planck }}=\left(\hbar G / c^{5}\right)^{1 / 2} \approx$ $10^{-43} \mathrm{~s}$, Planck length $L_{\text {Planck }}=c T_{\text {Planck }} \approx 10^{-35} \mathrm{~m}$ and Planck mass $M_{\text {Planck }}=\hbar /\left(c^{2} T_{\text {Planck }}\right) \approx$ $10^{-8} \mathrm{~kg}$. Experiments on quantum gravity require access to these scales. To access these scales directly using accelerators would require $10^{19} \mathrm{GeV}$ accelerators, well beyond any conceivable experiments. 
Nonetheless, there have been significant developments in examining possible signatures of quantum gravity. A number of phenomena have been proposed to be potentially observable, including 'deformations' of special relativity and 'imprints' in the cosmic microwave background. For recent reviews, see e.g. [4] and [5] and references therein.

One possible way of accessing the Planck scale is to use the concept developed by Einstein in his study of thermal fluctuations of small particles through the Brownian motion [6]. Modern experimental methods are so much in advance of those of Einstein's time, that we are now in a position to consider accessing the Planck scales by a method analogous to Brownian motion, a concept first pointed out by Ellis et al [7]. The curvature of spacetime produces changes in proper time, the time measured by moving clocks. For sufficiently short time intervals, near the Planck time, the proper time fluctuates strongly due to quantum fluctuations. For longer time intervals, proper time is dominated by a steady drift due to smooth spacetime. Proper time is therefore made up of the quantum fluctuations plus the steady drift. The boundary separating the shorter time scale fluctuations from the longer time scale drifts, is marked by a time $\tau_{0}=\lambda T_{\text {Planck }}$ that defines the borderline between semiclassical and fully quantum regimes of gravity, in terms of a dimensionless cut-off parameter $\lambda$.

Since the quantum to classical transition of gravity is expected to occur at a length scale $\lambda_{0}=$ $\lambda L_{\text {Planck }}$ larger than $L_{\text {Planck }}$ by a few orders of magnitude, the parameter $\lambda$ should approximately satisfy $\lambda>10^{2}$. Its actual value is model dependent. E.g. the extra-dimensional gravity model as per Arkani-Hamed et al has the fundamental scale of gravity in the $\mathrm{TeV}$ range [8], which would place $\lambda$ to be as large as around $10^{16}$. Below, the motion of a quantum wave packet will be examined on a length scale larger than $\lambda_{0}$ where the time-varying background metric is assumed to be well described as a classical field. Markopoulou et al have recently developed a theoretical framework where particles are described as 'noiseless subsystems' in a background independent manner [9. However, we assume that at an appropriate low-energy limit of such a theory, the classical picture of geometry will emerge.

Matter wave interferometers are ideal in measuring decoherence effects and will be able to put upper limits on quantum fluctuations which will help guide the theoretical work. An atom is a quantum clock with a very high frequency proportional to its mass. In contrast, the proper time intervals of massless particles like photons are unaffected as they travel along null geodesics. In an atom interferometer, an atomic wavepacket is split into two coherent wavepackets that follow different paths before recombining. The phase change of each wavepacket is proportional to the proper time along its path, resulting in constructive or destructive interference when the wavepackets recombine. The detection of the decoherence due to spacetime fluctuations on the Planck scale would provide experimental access to quantum gravity effects analogous to accessing to atomic scales provided by Brownian motion. A number of authors [10, 11, 12] have suggested investigating decoherence in the two-path atom interferometer where the separation of the wavepackets is large compared to the width of the wavepacket. There have also been studies of decoherence models using non-propagating conformal fluctuations of spacetime by Sanchez-Gomez [13, and using Newtonian gravity by Kay [14. 
Recent work by Power and Percival [10] considered a model of the 'conformal field' $A$ that conformally deforms the Minkowski spacetime metric $\eta_{\alpha \beta}=\operatorname{diag}(-1,1,1,1)$ (using $\alpha, \beta=0,1,2,3$ as spacetime coordinate indices) into the curved spacetime metric $g_{\alpha \beta}$ via

$$
g_{\alpha \beta}=(1+A)^{2} \eta_{\alpha \beta} .
$$

They assumed that the incoherent 'conformal waves' of $A$ are produced by their own quantum mechanical zero point fluctuations. These waves then interact with wavepackets of massive particles through the effective Newtonian potential using the weak field and slow motion approximation. The nonlinear contribution from conformal field causes a decoherence of the quantum wavepackets. The random walk of the conformal field is modelled by a Gaussian correlation function with $\tau_{0}$ as the correlation time. In terms of the density matrix $\rho$ of the wavepacket at time $t=0$ and its change due to spacetime fluctuations $\delta \rho$ at time $t=T$, the decoherence is represented by the loss of contrast of the form [10]:

$$
\frac{\delta \rho}{\rho(0)}=\sqrt{\frac{\pi}{2}} \frac{M^{2} c^{4} T A_{0}^{4} \tau_{0}}{\hbar^{2}}
$$

where $M$ is the mass of the atom and $A_{0}$ is the amplitude of the fluctuating $A$, assumed to be much less than unity. The conformal field is assumed to have frequency contributions from 0 up to a cut-off frequency $\omega_{0}=2 \pi / \tau_{0}$. Based on atom interferometer experiments using caesium atoms with $M=133 \mathrm{amu}$ separated for $T=0.32 \mathrm{~s}$ resulting in a loss of contrast of $\delta \rho / \rho(0)=3 \%$ [15], Power and Percival estimate the lower bound on $\lambda$ to be of order 10, outside the expected range.

Previous models [10, 11, 12] suffer from a number of serious drawbacks and are too crude to make predictions [16]. They suspend all but the conformal degrees of freedom of gravity at the cost of violating Einstein's equations. Consequently, most recent investigations of gravitational decoherence have focused on gravitational waves (GWs) of astrophysical and cosmological origins 17, 18. The smallness of these waves have, however, rendered the chance of detecting their decoherence effects very slim. Here we introduce for the first time new components of the gravitational field describing shearing actions on spacetime geometry, commonly referred to as the spin-2 GWs [19, 20]. These components define the conformal geometry of spacetime that yields the full geometry when combined with the conformal field. Careful initial data analysis reveals that the spin-2 GWs carry the true dynamical degrees of freedom of GR [21, just like the spin-1 EM waves carrying the true dynamical degrees of freedom of Maxwell's theory of electromagnetism. GWs are believed to be quantized into spin-2 gravitons and hence have zero point energy.

The essential requirement for the theoretical framework in which the conformal filed interacts with GWs at zero point energy is a conformally decomposed Hamiltonian formulation of GR. Such a theoretical framework has been established in recent papers [19, 20. It allows us to consider a general spacetime metric of the form

$$
g_{\alpha \beta}=(1+A)^{2} \gamma_{\alpha \beta} .
$$


in terms of the conformal field $A$ and the rescaled metric $\gamma_{\alpha \beta}$. We shall work in a standard laboratory frame where the direction of time is perpendicular to space. Accordingly, we set $\gamma_{00}=-1$ and $\gamma_{0 a}=0$ (using $a, b=1,2,3$ as spatial coordinate indices.) The spatial part of the metric $\gamma_{\alpha \beta}$ is denoted by $\gamma_{a b}$ and is normalized using $\operatorname{det}\left(\gamma_{a b}\right)=1$. Hence, $\gamma_{a b}$ will be referred to as the 'conformal metric' as it specifies the conformal geometry of space. It's inverse is denoted by $\gamma^{a b}$. The spacetime metric (3) therefore accommodates both the conformal field, as does the metric in (11), and in addition the spin-2 GWs encoded in the deviation of the conformal metric $\gamma_{a b}$ from the Euclidean metric $\delta_{a b}$.

Adopt, for a moment, units where $16 \pi G=c=1$. In Refs [19, 20, the canonical theory of general relativity has been constructed in terms of the conformal classes of spatial metrics by extending the Arnowitt-Deser-Misner (ADM) phase space consisting of the spatial metric $g_{a b}$ and its momentum $p^{a b},(a, b=1,2,3)$. The canonical transformation $\left(g_{a b}, p^{a b}\right) \rightarrow\left(\gamma_{a b}, \pi^{a b} ; \tau, \mu\right)$ is performed using a conformally transformed spatial metric $\gamma_{a b}$, its momentum $\pi^{a b}$, the scale factor $\mu=\sqrt{\operatorname{det} g_{a b}}$ and York's mean extrinsic curvature variable $\tau$ [21, 19]. The new canonical framework has the Hamiltonian constraint $\mathcal{H}$, diffeomorphism (momentum) constraint: $\mathcal{D}_{a}$ and conformal constraint $\mathcal{C}$ [20. In terms of these constraints, the Hamiltonian for gravity is given by [19, 20]:

$$
H=\int\left[N \mathcal{H}+X^{a} \mathcal{D}_{a}+Z \mathcal{C}\right] \mathrm{d}^{3} x
$$

using the lapse function $N$, shift vector $X^{a}$ and Lagrange multiplier $Z$ to effect the vanishing of the conformal constraint $\mathcal{C}$. We choose coordinate and scaling gauge conditions to facilitate the study of the coupling between the conformal and gravitational wave parts of gravity. In addition, these gauge fixings should allow our spacetime metric to be expressed in terms of a deviation from the Minkowski metric for ease of comparison with earlier approaches to the conformal field. We fix the conformal gauge by normalizing the conformal 3-metric according to $\operatorname{det}\left(\gamma_{a b}\right)=1$. The momentum $\pi^{a b}$ is then required to be traceless with respect to $\gamma_{a b}$. It is possible to find parameterizations to satisfy the above properties of $\gamma_{a b}$ and $\pi^{a b}$. However, their explicit construction is not required for our present analysis.

The normalized conformal 3-metric $\gamma_{a b}$ can be extended into a normalized conformal 4-metric $\gamma_{\alpha \beta}$ by adopting the Gaussian coordinate conditions for the time-time and time-space metric components $\gamma_{00}=-1$ and $\gamma_{0 a}=0$. Using the 'conformal field' $A$, we can relate the physical and conformal metrics simply by Eq. (3). This fixes the lapse function and shift vector to be $N=1+A$ and $X^{a}=0$ respectively, so that the direction of time is chosen as perpendicular to space. We then perform the canonical transformation $\left(\gamma_{a b}, \pi^{a b} ; \tau, \mu\right) \rightarrow\left(\gamma_{a b}, \pi^{a b} ; A, P\right)$ where $P$ is the momentum of $A$. In terms of these variables, the gravitational Hamiltonian (4) becomes

$$
H=\int\left[\mathcal{H}^{(\mathrm{CF})}+\mathcal{H}^{(\mathrm{GW})}\right] \mathrm{d}^{3} x
$$


where

$$
\mathcal{H}^{(\mathrm{CF})}=-\left(\frac{1}{24} P^{2}+6 \gamma^{a b} A_{, a} A_{, b}\right)
$$

is the Hamiltonian density for the conformal field and

$$
\mathcal{H}^{(\mathrm{GW})}=(1+A)^{-2} \pi_{a b} \pi^{a b}-(1+A)^{2} R_{\gamma}
$$

is the Hamiltonian density for the GWs, where $R_{\gamma}$ is the Ricci scalar curvature of $\gamma_{a b}$. Although we have adopted a canonical description, the covariance of our approach can be reaffirmed by means of a Legendre transformation on the Hamiltonian (5), yielding the action for gravity of the covariant form:

$$
S=\int\left[6 \gamma^{\alpha \beta} A_{, \alpha} A_{, \beta}+(1+A)^{2} \mathcal{R}_{\gamma}\right] \mathrm{d}^{4} x
$$

where $\mathcal{R}_{\gamma}$ is the Ricci scalar curvature of the 4-metric $\gamma_{\alpha \beta}$. In arriving at this action, we have used the momentum-velocity relations $\dot{\gamma}_{a b}=2(1+A)^{-2} \pi_{a b}$ and $\dot{A}=-P / 12$, where the overdot denotes a time derivative. By using the latter relation, we can eliminate the momenta in $\mathcal{H}^{(\mathrm{CF})}$ and write it, after restoring the full units, as

$$
\mathcal{H}^{(\mathrm{CF})}=-\frac{3 c^{4}}{8 \pi G}\left(c^{-2} \dot{A}^{2}+\gamma^{a b} A_{, a} A_{, b}\right) .
$$

This Hamiltonian density has a remarkable feature of being similar to that of a massless scalar field but with a 'wrong sign', i.e. negative energy density, which has important physical consequences to be explored below.

It is worth stressing that only physical degrees of freedom have zero point energies. In principle, 3 conditions can be used to eliminate the coordinate redundancy in the conformal metric $\gamma_{a b}$ containing 5 components. Assuming small deviation from the Minkowski spacetime, the vacuum energy due to $\mathcal{H}^{(\mathrm{GW})}$ in the semiclassical domain can be estimated by considering the quantization of weak GWs in a box of unit volume. In this case, the number of modes per frequency is given by

$$
n(\omega)=\frac{4 \pi \omega^{2}}{(2 \pi c)^{3}} .
$$

The energy density $\mathcal{H}_{0}^{(\mathrm{GW})}$ due to zero point energy gravitons with 2 polarizations and frequencies up to the cut-off value $\omega_{0}=2 \pi / \tau_{0}$ is

$$
\mathcal{H}_{0}^{(\mathrm{GW})} \approx 2 \int_{0}^{\omega_{0}} \frac{1}{2} \hbar \omega n(\omega) \mathrm{d} \omega=\frac{2 \pi^{2} c^{2}}{\lambda^{4}} \frac{M_{\text {Planck }}}{L_{\text {Planck }}^{3}}
$$

since each frequency mode has energy $\frac{1}{2} \hbar \omega$. For $\lambda>10^{2}$, the above $\mathcal{H}_{0}^{(\mathrm{GW})}$ value amounts to a vacuum mass density of $10^{68}-10^{84}$ metric tons per litre! However, we must also take 
into account the contribution from the conformal field, which can be determined using the Hamiltonian constraint to be:

$$
\mathcal{H}^{(\mathrm{CF})}=-\mathcal{H}_{0}^{(\mathrm{GW})} .
$$

The relation (11) admits a very simple picture: Every pair of gravitons having the same zero point energy $\frac{1}{2} \hbar \omega$ but two different helicities is accompanied by a 'quantum' of the conformal field with a compensating energy of $-\hbar \omega$. However, a further implication is that the resulting conformal field may be observable through an atom wave decoherence experiment. Since all components of the metric deviating from the Minkowski values are assumed to be small, the Newtonian approximation for the slow motion of a matter wave packet applies. However, in this limit, only the conformal field contributes to the effective Newtonian gravitational potential $-\left(g_{00}+1\right) / 2$ but not the GW fields. The amplitude of the fluctuating conformal field $A$ can be derived from the energy density condition (11) together with Eqs (8) and (10) in a mode-by-mode fashion as follows:

$$
\frac{3 c^{4}}{8 \pi G}\left\langle c^{-2} \dot{A}^{2}+|\nabla A|^{2}\right\rangle \mathrm{d} \omega=\hbar \omega n(\omega) \mathrm{d} \omega
$$

where $\langle\cdot\rangle$ denotes averaging over space and the vector modulus $|\cdot|$ is approximated using the Euclidean norm. Let $A(\omega)$ be the amplitude of $A$ at frequency $\omega$. Using relation (9), the above relation yields $\left\langle A(\omega)^{2}\right\rangle=2 T_{\text {Planck }}^{2} \omega / 3 \pi$ which shows a clear spectral distribution of the conformal field towards higher frequencies. From this, the overall amplitude squared for $A$ due to quantum vacuum fluctuations is

$$
A_{0}^{2}=\int_{0}^{\omega_{0}}\left\langle A(\omega)^{2}\right\rangle \mathrm{d} \omega=\frac{4 \pi}{3 \lambda^{2}} .
$$

Within the Newtonian approximation for the atom wave motion, we finally obtain an expression for the cut-off parameter $\lambda$ by substituting Eq. (12) into Eq. (2) to be

$$
\lambda=\left(\frac{8 \sqrt{2 \pi^{5}}}{9} \frac{M^{2} c^{4} T_{\text {Planck }} T}{\hbar^{2}(\delta \rho / \rho(0))}\right)^{1 / 3} .
$$

For the mentioned atom interferometer experimental data using caesium atoms with $M=133$ amu separated for $0.32 \mathrm{~s}$ resulting in a loss of contrast of about $3 \%$ [15, formula (13) yields a lower bound on $\lambda$ to be:

$$
\lambda \geq 7600 .
$$

The inequality counts for any other causes of decoherence. This lower bound is well within the expected range $\lambda>10^{2}$ for low energy quantum gravity.

For almost a century it has been widely perceived that the lack of experimental evidence for quantum gravity has presented, and will continue to present, a major barrier to its breakthrough. However, armed with the sensitivity of modern matter wave interferometers at the quantum level, the possibility of using a 'macroscopic' instrument to investigate Plank scale physics is now a real 
possibility. Following recently formulated conformal decomposition in full canonical gravity, we have developed a new approach to gravitational decoherence due to ground state gravitons and have demonstrated that the resulting conformal field can lead to observable effects by causing quantum matter waves to loss coherence.

Further improved measurement may decrease the upper bound of decoherence resulting in an increased $\lambda$. A space mission similar to the proposed HYPER atom wave interferometer can provide such improvements 22]. Advanced matter interferometers using molecules under active development [23, 24, 25] are designed to enhance decoherence effects using particles of larger mass. However, the additional decoherence effects due to the structure of a molecule must be controlled. Recent experiments using $\mathrm{C}_{70}$ fullerene molecules with $M=70 \times 12 \mathrm{amu}$, separated for $0.004 \mathrm{~s}$ result in a loss of contrast of $4 \%$ [25]. According to formula (13), this yields $\lambda \geq 5500$, close to the lower bound given in Eq. (14). For every 3 orders of magnitude improvement in the loss of contrast, there is only 1 order of magnitude change in the lower bound of $\lambda$. Therefore, its possible rise with any decrease of decoherence measurement is rather slow. This, combined with the fact that the lower bound of $\lambda$ in the range 5500-7600 calculated using current experimental data is already within the expected range $\lambda>10^{2}$, is a very good sign. It strongly suggests that the measured decoherence effects are converging towards the fundamental decoherence due to quantum gravity. The experimental determination of $\lambda$ will be compelling evidence for the quantum behaviour of spacetime and set a stringent benchmark in our search for the ultimate theory of quantum gravity.

\section{Acknowledgments}

We would like to thank M Sandford, G Amelino-Camelia, K Hornberger, B Kent, I C Percival and J W York for fruitful discussions. The work was supported by the CCLRC Centre for Fundamental Physics.

\section{References}

[1] Green M, Schwarz J and Witten E 1987, Superstring theory, (Cambridge University Press, Cambridge)

[2] Ashtekar A 1986, Phys. Rev. Lett. 57, 2244

[3] Rovelli C and Smolin L 1995, Phys. Rev. D 52, 5743

[4] AmelinoCamelia G, Lämmerzahl C, Macias A and Müller H 2005, Preprint gr-qc/0501053

[5] Smolin L 2006, Preprint hep-th/0605052

[6] Einstein A 1905, Ann.d.Physik, 17, 549 
[7] Ellis J, Hagelin J S, Nanopolous D V and Srednicki M 1984, Nucl. Phys. B 241, 381

[8] Arkani-Hamed N, Dimopoulos S and Dvali G 1998, Phys. Lett. B 429, 263

[9] Kribs D W and Markopoulou F 2005, Preprint gr-qc/0510052

[10] Power W L and Percival I C 2000, Proc. Roy. Soc. Lond. A 456, 955

[11] Percival I C and Strunz W T 1997, Proc. R. Soc. Lond. A 453, 431

[12] Power W L 1999, Proc. Roy. Soc. Lond. A 455, 991

[13] Sanchez-Gomez J L 1994, ed. L Diosi, G B Lukacs, World Scientific, Singapore

[14] Kay B 1998, Class. Quantum Grav. 15, L89

[15] Peters A, Chung K Y, Young B, Hensley J and Chu S 1997, Phil. Trans. R. Soc. Lond. A 355, 2223

[16] Amelino-Camelia G 2005, in Planck scale effects in astrophysics and cosmology, edited by G. Amelino-Camelia and J. Kowalski-Glikman (Springer, Berlin)

[17] Reynaud S, Lamine B, Lambrecht A, Neto P M and Jaekel M-T 2004, Gen. Rel. Grav. 36, 2271

[18] Lamine B, Hervé R, Lambrecht A and Reynaud S 2006, Phys. Rev. Lett. 96, 050405

[19] Wang C H-T 2005, Phys. Rev. D 71, 124026

[20] Wang C H-T 2005, Phys. Rev. D 72, 087501

[21] York J W 1972, Phys. Rev. Lett. 28, 1082

[22] HYPER, Hyper-precision cold atom interferometry in space, ed. Assessment study report ESA-SCI (2000).

[23] Hornberger K et al 2003, Phys. Rev. Lett. 90, 160401

[24] Hackermueller L et al 2003, Phys. Rev. Lett. 91, 090408

[25] Hackermueller L, Hornberger K, Brezger B, Zeilinger A and Arndt M 2004, Nature 427, 711 